\title{
Surface Modeling in the Course of Engineering Graphics
}

\author{
Tatiana Markova, Andrey Bochkov, Mikhail Kokorin, and Tatiana Nikitina \\ Peter the Great St. Petersburg Polytechnical University, 29 Polytechnicheskaya st., \\ St.Petersburg, 195251, Russia \\ markova_tvespbstu.ru, bochkov_alespbstu.ru, \\ kokorin_msespbstu.ru, nik_tatianalspbstu.ru
}

\begin{abstract}
Development of the designer's documentation package by means of CAD systems implies good skills with toolkits of solid and surface modeling. The ability to identify the surface type, its generation process and consequently to choose the optimal modeling is acquired in the descriptive geometry course, as well as engineering graphics and computer graphics courses. The article describes training assignments designed for students of machine building departments aimed both at consolidation of theoretical knowledge and development of skills required to identify and model various surface types.
\end{abstract}

Keywords: Descriptive Geometry, Engineering Graphics, Surface Modeling, CAD-systems, CAD KOMPAS-3D.

\section{Introduction}

Currently one of most advanced modeling techniques for designing 3D models of complicated shapes applied in CAD systems of higher and middle levels is surface modeling.

Surface and solid modeling are the two terms used to describe two methods of geometrical modeling of real objects. These methods have much in common: in both an engineer performs similar operations resulting in generating a shell which is a set of the object's surfaces joining at the edges that are crossing lines of these surfaces [1]. It is the sequence of operations that is different in the two methods. In solid modeling the process is performed with body shells possessing all attributes of the physical body, with the modeling process often replicating the object production process: first a comparatively simple shape is generated that gradually acquires the desired form as a result of additional operations. The obtained continuous shell is a set of the resultant body surfaces. In industry, models of the majority of products' parts can be obtained by means of solid modeling.

In surface modeling the part surfaces of the object to be modeled are generated at the first stage. Then the surfaces are intersected, cut at the crossing lines, converged by curving surfaces, etc, with the resulting shell assembled from these surfaces. It may not

Copyright (C) 2020 for this paper by its authors. Use permitted under Creative Commons License Attribution 4.0 International (CC BY 4.0). 
be continuous since often only the most complicated part of the object is modeled in such way, while the rest can be modeled by basic solid technique. Surface modeling enables a designer to generate an object of an arbitrary shape, therefore it is normally used in automobile, aviation and ship-building industries to design such components as hull, car bodies, cast and molded parts.

Operations and commands used for modeling in various CAD systems are known to have different names, yet in all cases the model generation process is a vivid illustration of practical applications of the surface generation theory taught in the courses of descriptive geometry and engineering graphics. Therefore, from the authors' perspective, computer technology of surface modeling can be incorporated in the curriculum along with theory studies.

\section{Theoretical basis of surface modeling techniques used in CAD systems}

Surface modeling as well as that of other associated geometrical objects, is studied in various sections of mathematics. Analytical and differential geometry, variational calculus, topology and computational mathematics serve the theoretical basis for geometrical modeling. Leaving beyond the scope of this article the great variety of tasks and problems involved in development of mathematical kernel of CAD systems we intend to focus on graphic techniques studied in the course of descriptive geometry and described in textbooks [2], as well as their correlation with techniques used by a modeler working in CAD systems.

There are two basic techniques used in descriptive geometry for surface modeling: kinematic and wireframe ones, rotation surfaces, ruled surface with one line and a vertex, ruled surface with plane of parallelism, helicoids are basic surfaces which can be generated by means of moving the generatrix according to a rule determined in certain way. Normally a graphically specified repere on the complex drawing is used, which is complemented by a verbal description of the generatrix movement. In teaching practice the wireframe method is paid much less attention to, primarily due to the lack of time allocated in the curriculum, though surfaces of numerous industrial products can be constructed only by this method. Thus it desirable to include the study of the so-called "key" designing techniques [3-4] in the discipline curriculum.

Russian system CAD KOMPAS-3D is used here to consider the basic operations of computer surface modeling, it being most widely used in courses of descriptive geometry in engineering departments of Russian universities. Toolkit "Wireframe and Surfaces" contains "Surfaces" panel with commands to be used to generate Extrusion Surfaces, Revolution Surface, Surface by Path, Lofted Surface, Ruled Surface by two directrixes or a line and a vertex, Surface by Point Layer, Surface by Grid of Curves and Surface by Grid of Points. A number of additional operations such as Patch, Surface Connection, Surface Equidistant Line, Knit Surface, Surface Trimming, Splitting Surface, Delete Faces, Surface Extension, Fillet and Champer are used to generate a final model from initial basic surfaces. 
To check the surface and interfaces smoothness, the smoothness check mode is used. When necessary, a solid body can be obtained from the continuous set of surfaces. It should be noted that surface names and terms used in CAD KOMPAS-3D system, actually like in other CAD systems do not always correlate with the ones accepted in mathematics. Thus, here surface repere is constructed in so-called Sketch or several Sketches. Then one of the above mentioned commands is applied to this sketch. However, it is always possible to trace the connection with the theory taught in descriptive geometry course.

The simplest example is a revolution surface. A flat projection model of such surface comprises two projections of straight revolution axis and the generatrix, which in a general case is any spatial or flat line. Since the same surface can be generated by revolution of various generatrixes the simplest, i.e. flat line is chosen. In generation of the 3D model of a revolution surface the following procedure is applied: first a surface is chosen, then its sketch is produced with a line of required shape, next, the revolution axis is specified, and finally shape generation operation command is applied.

Not only revolution surfaces, but also transition surfaces, tubular, canal surfaces, helicoids, ruled surfaces, as well as complex topographic surfaces are constructed in CAD systems according to algorithms appropriate for their determinants taught in descriptive geometry. Besides, many surfaces are known to be produced by different ways and correspondingly can be set by various determinants with various techniques and commands used for their modeling. The designer's task is to choose the most appropriate solution in each particular case.

\section{Descriptive Geometry as a Basic Discipline for Studying Computer 3-D Modeling Techniques}

Skills in development of computer geometric models which can serve the basis for preparation of the required design and technology documents, load calculation, structural improvements, technological processes simulation as well as software development for computer numerical control (CNC) machine tools, is one of the main requirements to the graduates from university engineering departments.

Not only the product development technology have changed, but also the content of design materials. This determines the necessity to adjust teaching methods for the disciplines of graphic cycle including descriptive geometry, engineering and computer graphics or integrated courses that are to substitute them. Problems of academic process organization are discussed in professional community [5-7], interesting ideas in teaching methodology and knowledge testing are suggested [8-15].

We agree with the opinion of some authors who point out to the importance of descriptive geometry as an academic discipline [5-7], however, taking into consideration the demands of digital economy concerning qualifications of new products designers we consider it reasonable to broaden the range of subjects taught in the engineering graphics course. From our perspective, teaching 3D modeling techniques is the task of graphics' teachers that should be carried out on the basis of descriptive geometry theories. It's specialists in the field of geometry and graphics possessing special knowledge 
and terminology, capable of tracing and exposing to students interrelations between computer modeling procedures and the discipline theory who can and should provide basic skills in CAD.

We suppose that for a number of reasons it is wise to teach descriptive geometry theory along with 3D modeling computer techniques as early familiarization with computer technologies is an increasingly growing demand. Besides, descriptive geometry is the discipline that lays foundations of spatial vision indispensable for computer modeling. Finally, it is in the descriptive geometry course normally taught in the first term that students get acquainted with shape generation theory, ways of surface identification, i.e. modeling in its broad sense.

It should be admitted that currently surface modeling problems are the most challenging and practically applicable ones, therefore curriculum should embrace a range of problems associated with their projection and computer modeling.

\section{Training Assignments for Formation of Surface Modeling Skills}

The teachers of Peter the Great St. Petersburg Polytechnic University have developed and tested in practice the first part of the assignment package aimed at comprehensive study of engineering graphics including descriptive geometry, engineering and computer graphics. It contains 8 exercises, which corresponds to the number of hours allocated for computer classes in the first term. Each exercise is a step-by-step instruction for performance of an assignment on a certain section of descriptive geometry, allowing at the same time to acquire skills with a certain set of computer tools. KOMPAS-3D CAD system is used as the system best adjusted to work in compliance with requirements of Unified Design Documentation System (UDDS) standards.

Each assignment starts with definition of its target and task, it also contains the performance and report sample; besides, it provides explanations how and why the required modeling is performed with reference to the relevant literature where this subject is considered in greater detail. The final part of the assignment contains tasks for selfpractice. It is recommended to do the analyzed task (step-by-step instruction) in the class under the teacher's guidance, which is to be consolidated later by performing a similar home assignment provided individually by the teacher to each student. A short survey of the assignments and description of their sections is to be found in articles [1617]. Here we would like to focus on two exercises devoted to surface modeling.

\subsection{Surface Modeling in KOMPAS-30 CAD System by Specified Determinant}

Evaluation of illumination and blur occurs on pairs of images similar to the face quality assessment in the baseline algorithm, with the use of Hinge Loss as $\mathrm{L}_{\text {Illumination }}$ and $\mathrm{L}_{\mathrm{Blur}}$. During training, the value of the loss function for pairs without markup is assumed to be zero. 
The exercise is to be performed alongside with study "Surface modeling" section in the descriptive geometry course. The task is formulated in the following way:

To design a surface model consisting of two parts determined by their repere, with one part being the continuation of the other and/or determining its domain of existence. A text document (*.doc) is to be submitted as a report containing surface reperes images, the designed model image as well as surfaces description and the list of the operations performed for their modeling.

The repere image is to be attached, their examples given in Fig. 1-4. Here and further the article contains the examples from the step-to-step instructions from the Assignment book and assignments performed by the students, where frontal projections are designated by index 1 , horizontal ones by index 2 , profile and other additional orthogonal projections by index 3 , while intersecting lines of projection planes (projection axes) are designated by letter $\mathrm{X}$ with the indices of the intersecting planes.

It should be noted that the task implies a certain degree of creativity: based on the given reperes of various surfaces can be designed by connecting components in different ways and choosing different dimensions. It is suggested to place one surface (any one) according to the given task, while the other one can be joined to it in an arbitrary way providing wherever possible a smooth transition from one component to the other one. Besides, when required or desired by the designer, either one or both surfaces can cut a part of the other reducing its spatial area. The surfaces designed according to the instructions described above can look for example, like this (see Fig. 5).
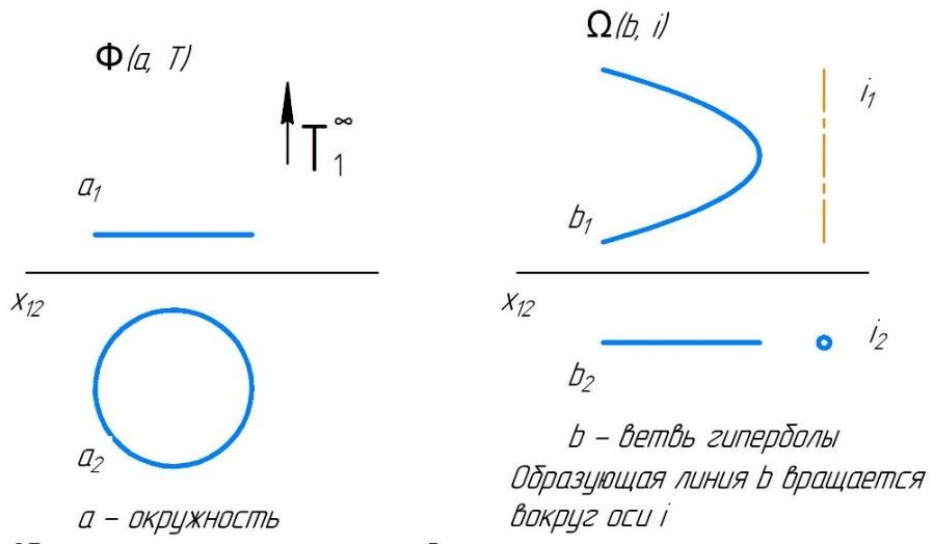

Образующая прямая пчния проходип

через вершину - несобственную

почку Т ч гкопьзип по напраВпяющечі

กUHUU $\square$

a)

(1)

Fig. 1. An example of the task for surface modeling. a - a circle. The straight generatrix passes through a vertex ideal point $\mathbf{T}$ ) and slides along the directrix $\mathbf{a} ; \mathbf{b}$ - a hyperbole branch. The generatrix $\mathbf{b}$ revolves around axis $\mathbf{i}$. 
6 T. Markova, A. Bochkov, M. Kokorin, T. Nikitina

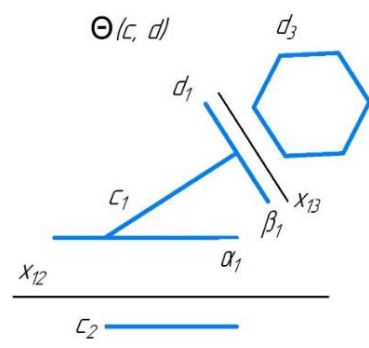

d - правипьныӥ шестицгопьниик Образующая пиния व перемещаепся ВӘоль напраВляющей пинич с.

Поверхность ограничена фронтальнопроецирующими плоскоспями снизу $-\alpha$, сверху $-\beta$

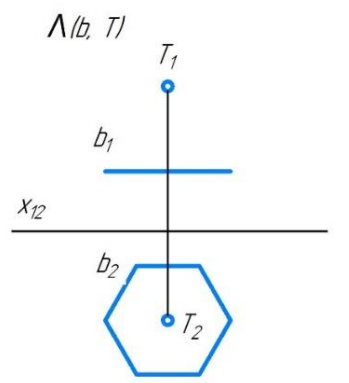

ь - праВильныш̈ шестиугольник ОБразующая прямая пиния проходит через Вершину -

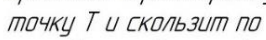
напраВляющеபі пUнUи 6

a)

Fig. 2. An example for surface modeling task. a - a regular hexagon. The generatrix d moves along the directrix .The surface is constrained by front projection planes: bottom $-\boldsymbol{\alpha}$, above $-\boldsymbol{\beta}$; $\mathbf{b}-$ a regular hexagon. The straight generatrix passes through a vertex $\mathbf{T}$ and slides along the directrix $\mathbf{b}$
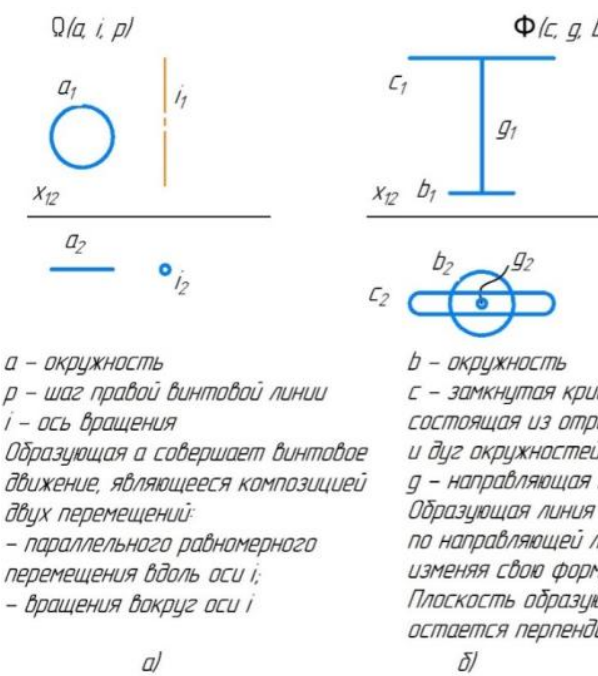

b - окрухность

ᄃ - замкнутпя кривая пиния

состоящая из отрезков прямых

4 ठуг окрухностей

g- направпяющая прямая пиния

Образующая пиния перемещается

по направпяющей пинии g. ппавно

чзменяя СВою форму оा Ь $K$ C.

Ппоскость образующей пинич

ocmaemcя перпендикулярной направпяющецे

ठ)

Fig. 3. An example for surface modeling task. $\mathbf{a}-$ a circle, $\mathbf{p}-$ a right-hand helix, $\mathbf{i}-$ a revolution axis. The generatrix a executes a helical movement which is a composition of two movements: parallel movement along axis $\mathbf{i}$ and revolution round axis $\mathbf{i}$. $\mathbf{b}-$ a circle, $\mathbf{c}-$ a closed curve consisting of straight pitches and circle arcs, $\mathbf{g}$ - a straight directrix. The generatrix line moves along the directrix $\mathbf{g}$ smoothly changing its shape from $\mathbf{b}$ to $\mathbf{c}$. The generatrix plane remains perpendicular to the directrix. 


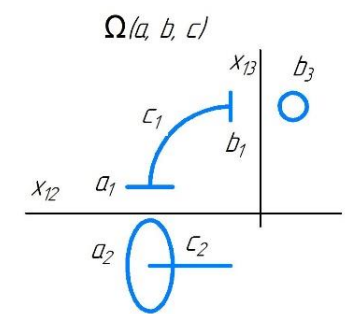

$$
\begin{aligned}
& \text { a- 3กกบก } \\
& \text { b - OKDYXHOCMь }
\end{aligned}
$$

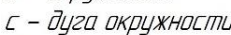

$$
\begin{aligned}
& \text { ОБразующая пиния перемещаетгя } \\
& \text { по направпяющей пинич с, ппавно } \\
& \text { Чзменяя СВОю ФормЧ оा व K } \\
& \text { Плоскость образцющеபі линши } \\
& \text { В кахDОм полохенUU огтаепся } \\
& \text { перпендикцлярной напраВпяющеці. }
\end{aligned}
$$$$
\text { a) }
$$

$\Theta(m, n)$
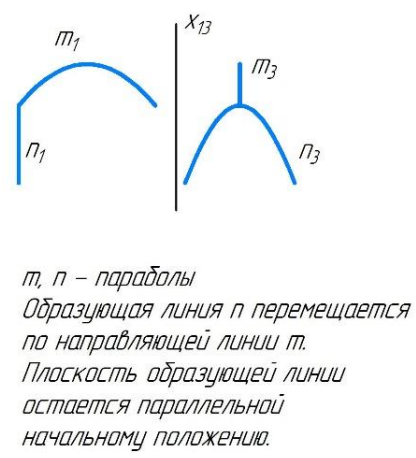

Fig. 4. An example of the task for surface modeling. $\mathbf{a}-$ an ellipse, $\mathbf{b}-$ a circle, $\mathbf{c}-$ an arc. The generatrix moves along the directrix $\mathbf{c}$ smoothly changing its shape from $\mathbf{a}$ to $\mathbf{b}$. The generatrix plane remains perpendicular to the directrix in every position. $\mathbf{m}, \mathbf{n}-$ parabolas. The generatrix $\mathbf{n}$ moves along the directrix $\mathbf{m}$. The generatrix plane remains parallel to the initial position.

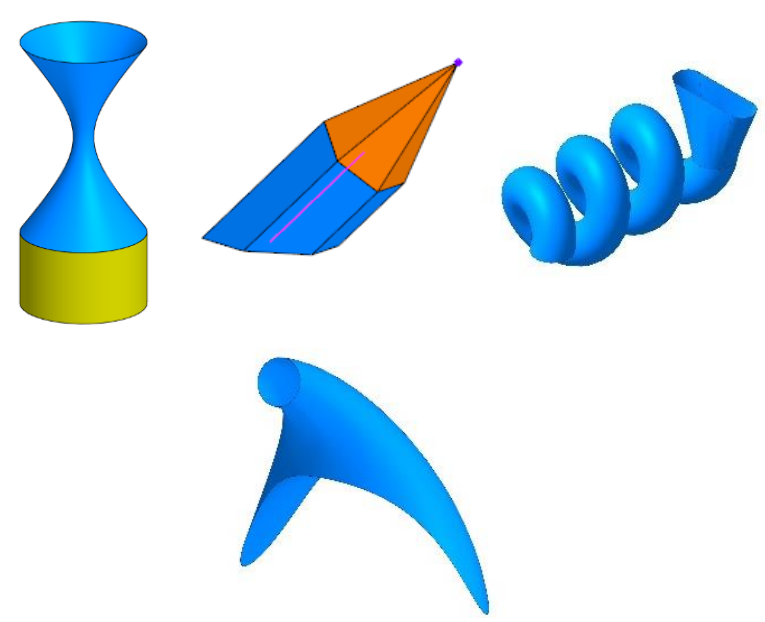

Fig. 5. Models of surfaces constructed according to tasks of Fig. 1-4.

The main goal of the assignment is to show the students how surface construction algorithms studied in the course of descriptive geometry are realized in practice, and besides, to get them acquainted with KOMPAS-3D line modeling tools including conic 
sections, spatial polygonal lines, cylindrical and conic helixes. This task is the third one in the methodological complex and here students for the first time are introduced to a new type of document referred to as Part, having learned flat drawing (2D) commands in KOMPAS-Graphic in the previous study stage. Thus, a gradual transition to the study of principles and approaches of 3D modeling is achieved.

The acquired knowledge and skills can be applied by students in the next, fourth assignment.

\subsection{Object Surface Modeling by the Given Image}

In this assignment a more sophisticated task is set: it is necessary to analyze the object's geometry (a machine part, a household object, a building component, etc.) based on its image (a photo, a picture), if necessary, to break it down into several geometrical objects, to identify the surface types, to understand how these surfaces are produced, to set graphical determinants, and finally to design the object's 3D surface model according to them .The task is formulated in the following way:

To identify and describe the surface types of the depicted object, to develop its 3D surface model. As a report a document Drawing is to be containing the repere (reperes) image with dimensions, the line designations, description of its construction method; to insert the photo of the modeled object as well as the screenshots of the model, sketches and construction tree.

The examples of the objects for modeling are presented in Fig.6. Here are both quite simple and rather complex objects, however, in all cases we tried to choose the objects with two types of surfaces at the most that may intersect. Our experience showed that not all students found this task easy: without the teacher's assistance not everyone was able to identify the surface type, to analyze its generation method, while differentiate between the lines that can be taken as generatrix or diretrix and others formed by intersecting surfaces; to specify the graphic determinants. It is not always easy to choose the optimal determinant and thus its modeling method even for a seemingly simple object.

Nevertheless, the assignment has a considerable practical importance; students practice identification of surface types of the objects in our environment, learn to specify reperes, to choose tools necessary for their modeling. We also draw students' attention to the fact that the term "Modeling" should not be treated only as the process of 3D model designing in CAD system. The ability describe the surface, to specify its determinant, to construct its flat projection model is not the least but probably the most important ability of the future engineer, since these skills enable him/her to adequately apply any tool of design documentation development, to work in any CAD system.

Besides, we would like to emphasize that any surface can be modeled in various ways and the goal is to choose the most suitable solution, with the shortest possible Model Tree and the simplest, however, completely defined sketches, therefore we try to show which commands should be preferably used, what are the opportunities of parameterization application in sketch preparation. Thus, the assignment goal is achieved: learning how to apply theoretical knowledge for product surface modeling.

Fig. 7 shows some models developed by students. For each model the document Tree is shown, it is seen that the least number of operations is used, the commands realizing 
the algorithms for construction of a certain surface type are chosen, whose name and description students provide in their report. Fig.8 shows the fragment of the report including repere constructed in two projections and the sketch for revolution surface generation of the vase.
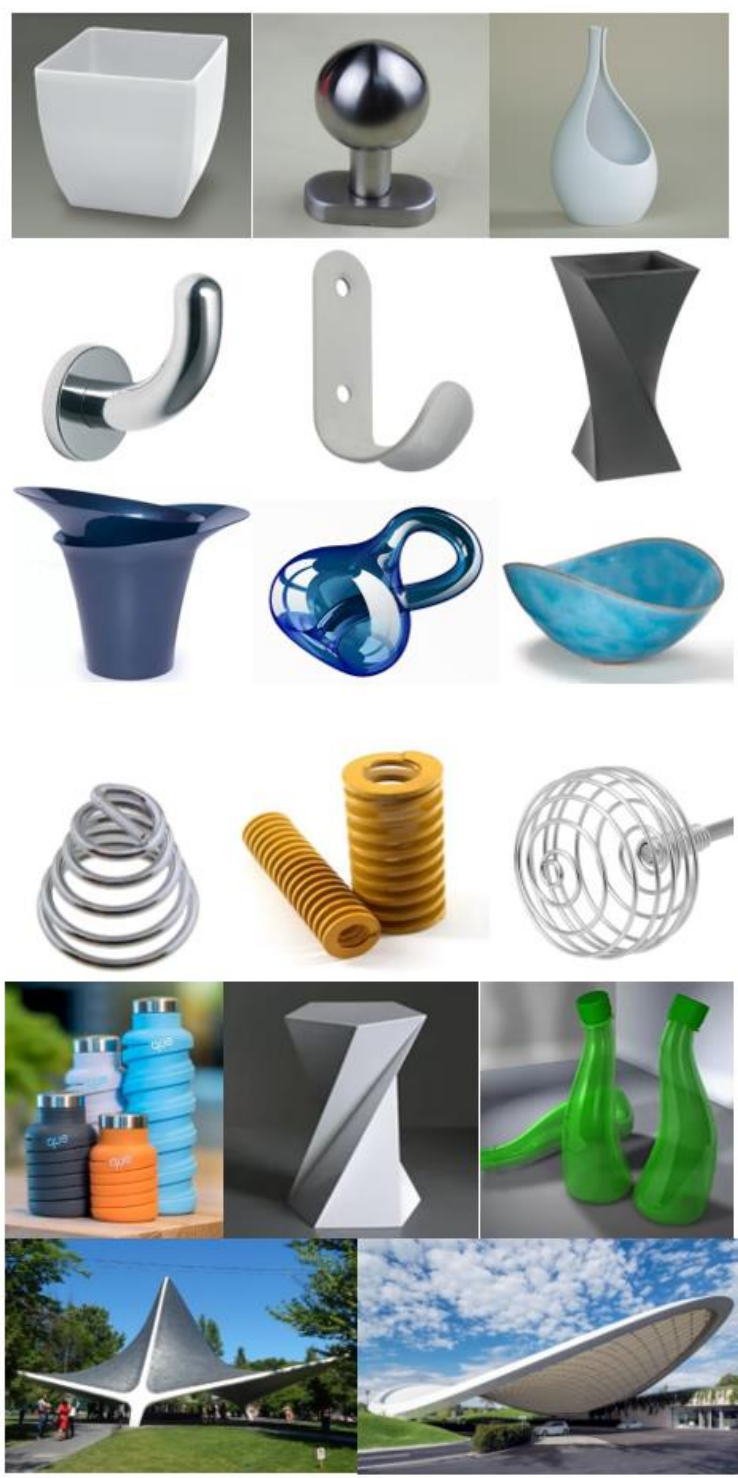

Fig. 6. Examples of tasks for object surface modeling by its image 
10 T. Markova, A. Bochkov, M. Kokorin, T. Nikitina
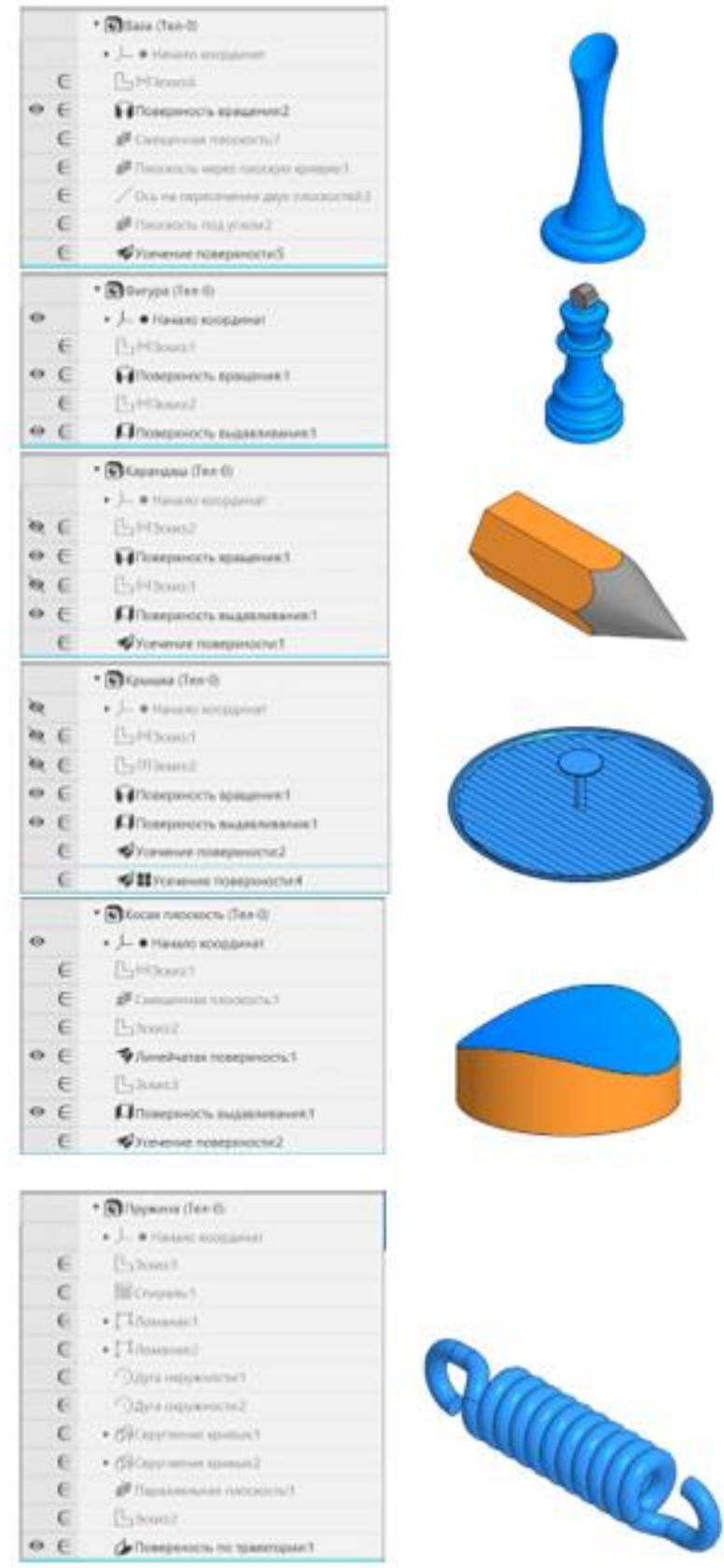

Fig. 7. Models of surfaces constructed by objects' images 

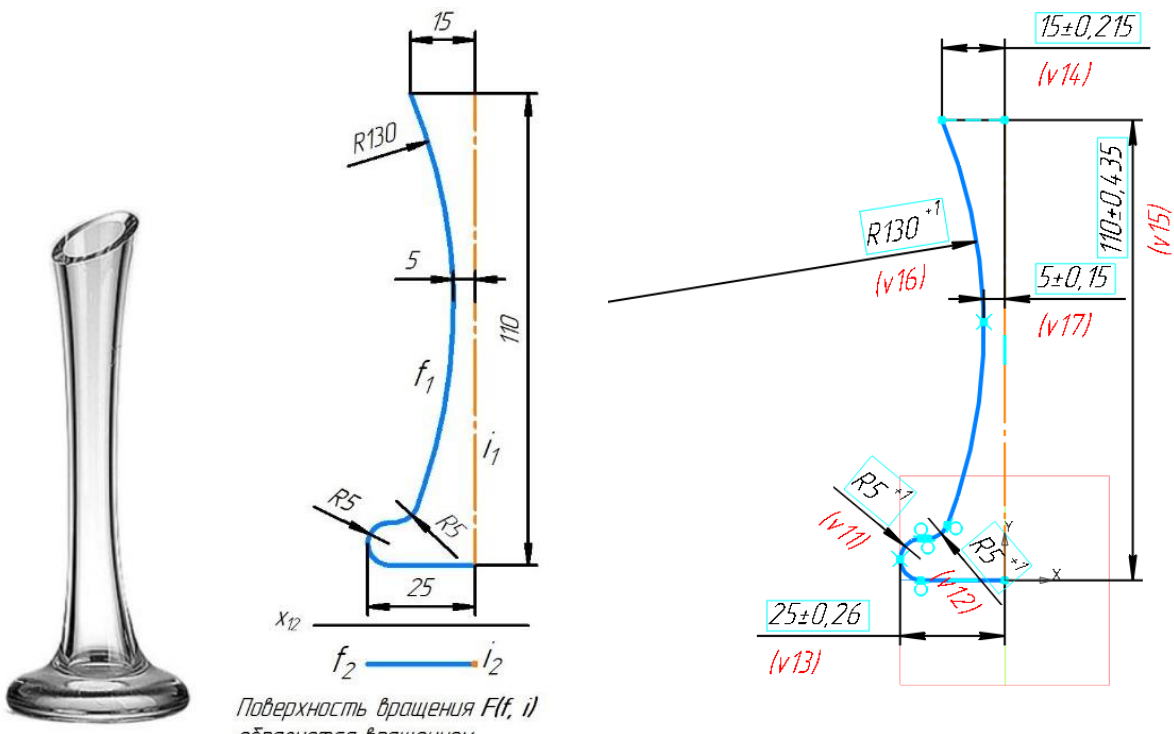

ооразуептя вращением

оорразующей пинич f Вокруг оси i

Fig. 8. Fragment of the report containing the image of the surface repere and the sketch for surface modeling of the vase revolution in CAD KOMPAS-3D. Revolution surface $\mathbf{F}(\mathbf{f}, \mathbf{i})$ is generated by revolution of generatrix $\mathbf{f}$ round axis $\mathbf{i}$.

We would like to pay attention to another important methodological aspect of the assignment: the sketch is parametrical, therefore specifying dimensions and imposing constraints (verticality, horizontality, tangency, point coincidence, etc.), controlling the objects' degrees of freedom and thus achieving complete precision, students acquire experience in shape geometry analysis, determination of required and sufficient number of the shape parameters and their optimal choice, which is directly connected with putting the part dimensions on its projection images (in the drawing) and will be useful later in studying the rules of correct drawing generation.

\section{Conclusion}

Performing the assignments described above students see that objects in their environment can be represented as a set of several solids, with surfaces subject to geometrical description. We suppose such analytical experience is important to understand descriptive geometry theory and the possibilities of practical application of the acquired knowledge, as well as in later studies of disciplines associated with industrial design.

It should be noted that the assignments have a rather high degree of complexity, which encourages students to develop initiative, independence and creativity, to form competences required for a modern engineer. The assignments are a part of methodological complex that implements an innovative integrated approach to teaching descriptive geometry, engineering and computer graphics. 


\section{References}

1. Golovanov, N.N.: Geometric Modeling. DMK Press, Moscow (2020)

2. Ivanov, G.S .: Descriptive Geometry: a textbook. Moscow State University, Moscow (2012)

3. Voloshinov, D.V: Designing Geometric Modeling. Theory, Practice, Automation. Lambert Academic Publishing, Saarbrucken (2010)

4. Voloshinov, D.V. Scientific Basis and Design Practice. Theory and Methods of Geometric Modeling: textbook. Training course. Publishing house Polytechnic. University, St. Petersburg (2008)

5. Salkov, N.A.: Place of Descriptive Geometry in the System of Geometric Education of Technical Universities. Geometry and Graphics. v. 4, No. 3, p. 53-61 (2016)

6. Stolbova, I.D., Aleksandrova, E.P., Kochurova, L.V., Nosov, K.G.: Profile Aspects of Graphic Education in a Polytechnic University. Higher education in Russia. v. 28, No. 3, p. $155-166$ (2019)

7. Tikhonov-Bugrov, D.E., Abrosimov, S.N., Dyumin, V.A.: Basic Principles of Engineering and Computer Graphics Teaching to Future Professionals. In: Proceedings of the International Conference on Computer Graphics and Vision "Graphicon" No. 28, p. 389-392 (2018)

8. Chempinsky, L.A.: Competencies Formation in the new Educational Course "Basics of Geometric Modeling in Mechanical Engineering". In: Problems of Graphic Training Quality of Students in a Technical University: Traditions and Innovations. v. 1, p. 303-307 (2019)

9. Khmarova, L.I., Usmanova, L.I.: The Application of Computer Technology in the Study of Graphic Disciplines. Bulletin of the South Ural State University. Series: Education. Pedagogical Sciences, v. 6, No. 2, p. 59-64 (2014)

10. Krasilnikova, G.A., Markova, T.V.: Knowledge of the Shape Geometry and Components Positioning Ratio in Engineered Product within 3D Self-testing Modeling Tool. Modern Engineering. No. 5, p. 90-97 (2016)

11. Markova, T.V.: Sketch As Evaluation Criterion And Means Of Formation Of Skills Of The Analysis And Synthesis Of Spatial Forms. In: Quality Problems of Graphic Training : Traditions and Innovations. v.1, p. 250-256 (2019)

12. Markova, T.V., Nikitina T.A.: On The Question Of Graphical Culture Formation Of A Technical University Student. Modern Engineering. Science and Education. No. 8, p. 48-62 (2018)

13. Ignatiev, S.A., Moroz, O.N., Tretyakova, Z.O., Folkin, A.I.: Experience in the Development of E-Learning Tools for Teaching Geometric-Graphic Disciplines. Geometry and Graphics. v. 5, No. 2, p. 84-92 (2017)

14. Boykov, A.A., Fedotov, A.M.: Implementing tools of the automatically verifying of the solutions of engineering geometry constructive problems to CAD. In: Proceedings of the International Conference on Computer Graphics and Vision "Graphon". No. 29, p 172-175 (2019)

15. Kheifets, A.L., Loginovsky, A.N., Butorina, I.V., Vasilyeva, V.N.: Engineering 3D Computer Graphics. Study Course for Bachelors of Science. 2nd ed., Moscow (2016)

16. Markova, T.V., Bochkov, A.L.: CAD as the Basis for the Integration of Geometric-graphic Disciplines. In: Proceedings of the Conference Innovative Technologies in Engineering Graphics: Problems and Prospects. April 24, 2020 Republic of Belarus, Brest, Russian Federation, Novosibirsk, NGASU (Sibstrin) (2020)

17. Markova, T.V.: Stimulating the Cognitive activity of Students in the Course of Engineering Graphics. In: Proceedings of the Conference Innovative Technologies in Engineering Graphics: Problems and Prospects. April 24, 2020 Republic of Belarus, Brest, Russian Federation, Novosibirsk, NGASU (Sibstrin) (2020) 